\title{
Correction to: The effect of teprenone on the intestinal morphology and microbial community of Chinese sea bass (Lateolabrax maculatus) under intermittent hypoxic stress
}

\author{
Hong-biao Dong • Yong-xu Sun • Ya-fei Duan • \\ Hua Li • Yong Li • Qing-song Liu • \\ Wen-hao Wang • Jia-song Zhang \\ Published online: 10 October 2020 \\ (C) Springer Nature B.V. 2020
}

\section{Correction to: Fish Physiol Biochem (2020) 46:1873-1882 \\ https://doi.org/10.1007/s10695-020-00838-0}

In the end of the Discussion section, following information should be included:

Conflict of interest: The authors declared no conflict of interest.

The online version of the original article can be found at https://doi.org/10.1007/s10695-020-00838-0

H.-b. Dong · Y.-f. Duan · H. Li · Q.-s. Liu •

J.-s. Zhang $(\bowtie)$

Fisheries Engineering Institute, Key Laboratory of South China Sea Fishery Resources Exploitation \& Utilization, Ministry of Agriculture and Rural Affairs, South China Sea Fisheries Research Institute, Chinese Academy of Fishery Sciences, No. 231 West

Xingang Road, Haizhu District, Guangzhou 510300, China

e-mail: donghongbiao@163.com

Y.-x. Sun

State Key Laboratory of Marine Environmental Science, College of Marine and Earth Sciences, Xiamen University, Xiamen, China

Y. Li

Zhuhai Modern Agricultural Development Center, Zhuhai,

Guangdong, China

-h. Wang

College of Fisheries and Life Science, Shanghai Ocean University, Shanghai, China
Funding information: Central Public-interest Scientific Institution Basal Research Fund, South China Sea Fisheries Research Institute, CAFS [2017YB15]; The fund of Guangzhou science and technology planning project [201904010169]; The Fund of Key Laboratory of South China Sea Fishery Resources Exploitation \& Utilization, Ministry of Agriculture and Rural Affairs, P. R. China [FREU2018-02]; Guangdong Modern Agricultural Industrial System Technology Innovation Team Project [2019KJ150].

Publisher's note Springer Nature remains neutral with regard to jurisdictional claims in published maps and institutional affiliations. 